\title{
INFLUENCE OF SUTURE TECHNIQUE AND SUTURE MATERIAL SELECTION ON THE MECHANICS OF END-TO-END AND END-TO-SIDE ANASTOMOSES
}

Norbert Baumgartner, MD

Philip B. Dobrin, MD, PhD

Mark Morasch, MD

Quan-Sheng Dong, MD

Robert Mrkvicka, BS
Experiments were performed in dogs to evaluate the mechanics of 26 end-to-end and 42 end-to-side artery-vein graft anastomoses constructed with continuous polypropylene sutures (Surgilene; Davis \& Geck, Division of American Cyanamid Co., Danbury, Conn.), continuous polybutester sutures (Novafil; Davis \& Geck), and interrupted stitches with either suture material. After construction, the grafts and adjoining arteries were excised, mounted in vitro at in situ length, filled with a dilute barium sulfate suspension, and pressurized in $25 \mathrm{~mm} \mathrm{Hg}$ steps up to $200 \mathrm{~mm} \mathrm{Hg}$. Radiographs were obtained at each pressure. The computed cross-sectional areas of the anastomoses were compared with those of the native arteries at corresponding pressures. Results showed that for the end-to-end anastomoses at $100 \mathrm{~mm} \mathrm{Hg}$ the cross-sectional areas of the continuous Surgilene anastomoses were $70 \%$ of the native artery cross-sectional areas, the cross-sectional areas of the continuous Novafil anastomoses were $90 \%$ of the native artery cross-sectional areas, and the cross-sectional areas of the interrupted anastomoses were $107 \%$ of the native artery cross-sectional areas $(p<0.05)$. At physiologic pressures, there were no differences in compliance among the three types of anastomosis. These data suggest that when constructing an end-to-end anastomosis in a small vessel, one should use an interrupted suture line or possibly continuous polybutester suture. Forty-two end-to-side anastomoses demonstrated no differences in crosssectional areas or compliance for the three suture techniques. This suggests that, unlike with end-to-end anastomoses, when constructing an end-to-side anastomosis in patients any of the three suture techniques may be acceptable. (J Thorac Cardiovasc Surg 1996;111:1063-72)
C ardiovascular surgeons are often called on to construct end-to-end or end-to-side anastomoses. The suture material selected and the surgical technique employed can influence the size and distensibility of the anastomotic lumen. ${ }^{1}$ Construction of vascular anastomoses with continuous sutures can be performed more rapidly and with less blood loss than construction with interrupted sutures, but a continuous suture line may cause the

From Loyola University Medical Center, Department of Surgery, Maywood, Ill., and Hines Veterans Administration Hospital, Hines, Ill.

Sutures studied were provided by Davis \& Geck, Co., Division of American Cyanamid Co., Danbury, Conn.

Received for publication Jan. 4, 1995.

Accepted for publication June 12, 1995.

Address for reprints: Philip B. Dobrin, MD, PhD, Loyola University Medical Center, Department of Surgery, 2160 S. First Ave., Maywood, IL 60153.

12/1/67029 anastomosis to behave as a region of focal constriction. This can cause turbulence and, in severe cases, produce a gradient across the anastomosis and decrease the flow provided to the distal vascular bed. ${ }^{2,3}$ Flow disturbances may dispose toward the development of intimal hyperplasia or thrombosis. ${ }^{4,5}$ Both the type of anastomosis and the suture material used may be important. Cardiovascular surgeons frequently use polypropylene sutures (Prolene; Ethicon, Inc., Somerville, N.J.; Surgilene; Davis \& Geck, Division of American Cyanamid Co., Danbury, Conn.) or expanded polytetrafluoroethylene sutures.* These suture materials are nonabsorbable and quite stiff. Another nonabsorbable suture used by some surgeons is polybutester (Novafil; Davis \& Geck). This suture material exhibits extensibility because of its copolymer composition. ${ }^{6,7}$

\footnotetext{
${ }^{*}$ Gore-Tex sutures, registered trademark of W.L. Gore \& Associates, Inc., Newark, Del.
} 
This article describes experimental studies comparing effects on the luminal dimensions and compliance of vascular anastomoses of the use of continuous versus interrupted suture techniques and of stiff (polypropylene) versus compliant (polybutester) suture material. Both end-to-end and endto-side anastomoses were examined in dogs in the short-term, with reversed femoral vein used as a vascular graft.

\section{Methods}

Mechanical properties of suture materials. Eight $1 \mathrm{~cm}$ long segments of 6-0 polypropylene (Surgilene) and 6-0 polybutester (Novafil) were studied in vitro. One end of the suture segment was secured to a Grass FT-10 force transducer (Grass Instruments, Quincy, Mass.) and the other end was wound about a cylindrical shaft $2.03 \mathrm{~mm}$ in diameter. The shaft was turned by a motor at a rate of $0.0025 \mathrm{~cm} / \mathrm{sec}$, producing a load increment of $1.48 \mathrm{gm} / \mathrm{sec}$ on the suture. The force exerted on the suture was recorded by the force gauge. The length that the suture was extended was determined by recording the number of turns of the shaft. Both polypropylene (Surgilene) and polybutester (Novafil) sutures were provided by Davis and Geck, (Division of American Cyanamid Co., Danbury, Conn.).

End-to-end anastomoses. Mongrel dogs 25 to $35 \mathrm{~kg}$ in weight were given general anesthesia and were prepared and draped for surgery. Incisions were made in the neck and the groins to expose the carotid and femoral vessels. A $4 \mathrm{~cm}$ segment of the carotid artery was excised. A 4 to $5 \mathrm{~cm}$ segment of femoral vein was excised, rinsed in heparinized saline solution at room temperature, and immediately implanted as a reversed vein interposition graft under no tension. Two stay sutures were placed 180 degrees apart at each anastomosis to hold the vessels in opposition with no tension. Anastomoses were constructed with continuous 6-0 polypropylene, continuous 6-0 polybutester, or interrupted 6-0 polypropylene or polybutester. A different suture material (polypropylene or polybutester) was used at each end of the vein-artery anastomoses. Bites were taken $0.5 \mathrm{~mm}$ from the edge of the vessels, and the suture line was advanced in $1 \mathrm{~mm}$ steps.

The interrupted anastomoses were constructed by placing diametrically opposed stay sutures. Interrupted sutures were then placed $1 \mathrm{~mm}$ apart. Each stitch was tied firmly. The continuous anastomoses were constructed by first placing two sutures 180 degrees apart. These were tied firmly and used to construct the continuous suture lines. The running suture lines were tied at these two diametrically opposed sites. During construction of the continuous anastomoses, just sufficient tension was applied to keep the vein apposed to the artery. In several continuous anastomoses, the actual force applied to the suture was measured with a detachable friction-grip device connected to a Grass FT-03 force gauge (Grass Instruments). This friction-grip device consisted of a plastic cone that could be inserted into and removed from a similar plastic cone. The suture was inserted between the two plastic cones and gripped by friction, permitting measurement of the force applied to the suture as the surgeon pulled the filament up and coapted the edge of the artery to the vein. The suture was removed from the friction grip device to permit application of the next stitch in the construction of the anastomosis. In the anastomoses measured, the force applied to 37 polypropylene suture bites was $19.7 \pm 1.3 \mathrm{gm}$ (mean \pm standard error of the mean) and the force applied to 37 polybutester suture bites was $8.7 \pm 0.5 \mathrm{gm}$. These forces were required to draw the suture through the tissue, take up the slack in the filament, and coapt the artery and vein graft. Inspection demonstrated that the difference in tension between the two sutures was that required to take up the slack and straighten the filaments as they were drawn through the tissues. Polypropylene is much stiffer than slack or slightly extended polybutester. The artery and vein graft were closely approximated and did not require tension to hold these vessels together.

After construction of the anastomoses, the grafts were perfused for 30 minutes in vivo with pulsatile arterial pressure. The graft and the adjacent arteries were then excised. The arteries at each end were cannulated with polyethylene catheters, and the vessels were mounted in a tissue bath at in situ length for mechanical studies. The bath was filled with normal saline solution held at $37^{\circ} \mathrm{C}$. The excised vessels were pressurized in $25 \mathrm{~mm} \mathrm{Hg}$ steps up to $200 \mathrm{~mm} \mathrm{Hg}$. After several stepwise cycles to relax the vessels, the lumen was filled with a dilute suspension of barium sulfate and the vessels were again pressurized in $25 \mathrm{~mm} \mathrm{Hg}$ steps up to $200 \mathrm{~mm} \mathrm{Hg}$. Dental films were placed immediately beneath the vessels and a dental radiographic machine was used to obtain radiographs of the vessels and the anastomoses at each pressure. The radiographs were projected at $\times 22$ magnification and traced onto paper. The accuracy and magnification of the radiographs was evaluated by obtaining radiographic images of stainless steel tubes that were cannulated and mounted in the tissue bath as though they were arteries. These steel tubes were measured with a mechanical micrometer. When the radiographs of the steel rods were projected at $\times 22$ magnification and were measured, the rods were found to be $103 \%$ of their dimensions as determined by direct measurement with a micrometer. The radiographic method was therefore judged to be sufficiently accurate for measuring the dimensions of the vessels. The diameters of the arteries, anastomoses, and vein grafts were measured on the projected images every $0.5 \mathrm{~mm}$ of real distance proximal and distal to the anastomoses. The cross-sectional area (CSA) of the endto-end anastomoses was calculated as $\pi \mathrm{r}_{\mathrm{i}}^{2}$, where $r_{i}$ is the internal radius of the lumen of the anastomoses. The compliance of the end-to-end anastomoses was calculated at each location for each pressure step by means of the following formula:

$$
C=\frac{\Delta D}{\Delta P \times D}
$$




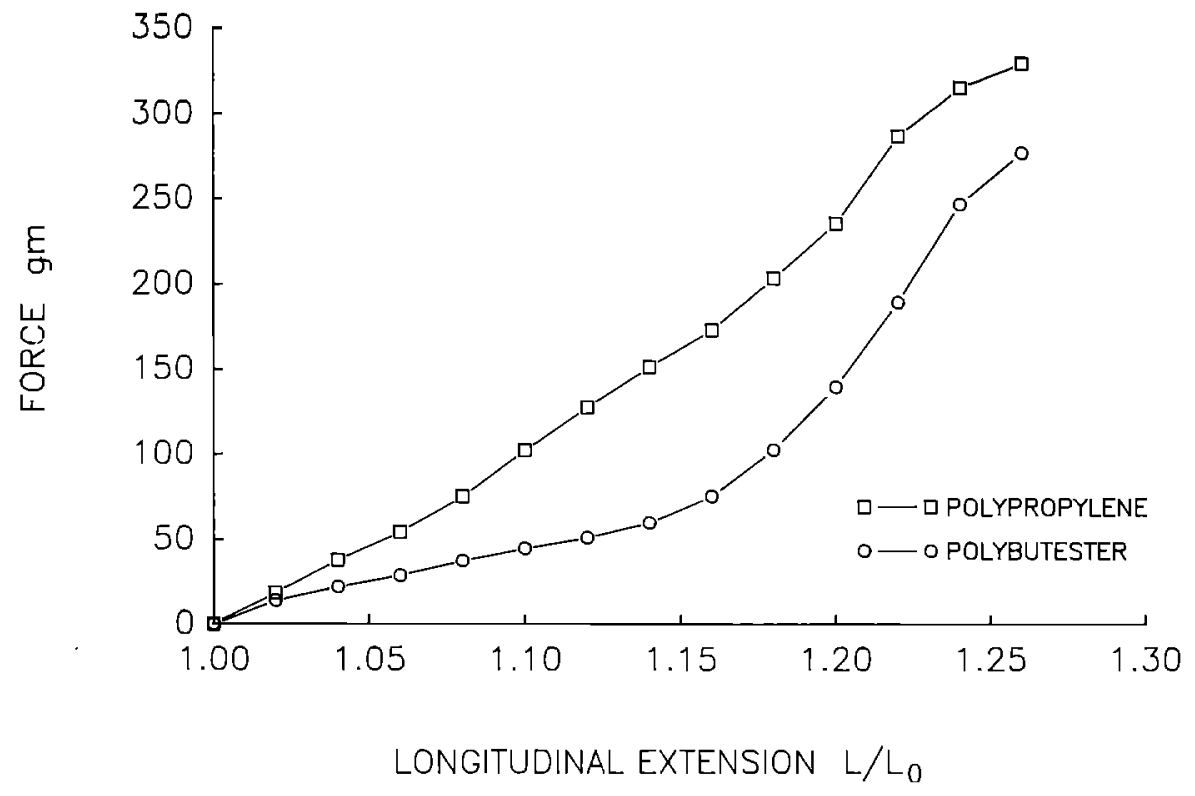

Fig. 1. Length-force curves of 6-0 polypropylene and polybutester sutures. Curves demonstrate extensibility of polybutester at deformations up to $15 \%$. Slopes are proportional to stiffness. $L / L_{o}$, Observed length divided by original length.

where $C$ is compliance, $\Delta D$ is the step change in diameter, $\Delta P$ is the step change in pressure, and $D$ is the lower, or "diastolic," diameter recorded for each pressure step.

End-to-side anastomoses. Mongrel dogs were anesthetized and prepared as described for end-to-end anastomoses. The femoral veins were excised bilaterally, rinsed with heparinized saline solution, reversed, and anastomosed as end-to-side bypass grafts. The ends of the vein were anastomosed to the side of the proximal and distal femoral artery. The bypassed region of femoral artery was ligated. Anastomoses were constructed with continuous 6-0 polypropylene, continuous $6-0$ polybutester, or interrupted 6-0 polypropylene or polybutester sutures. Continuous anastomoses were constructed with two sutures. One was placed at the heel of the graft, tied, and run along one side of artery-vein anastomosis. Bites $0.5 \mathrm{~mm}$ in size were taken from the edge of the artery and the vein, and the suture line was advanced in $1 \mathrm{~mm}$ steps. During construction, just sufficient tension was applied to the suture to keep the vein apposed to the artery. The suture was tied to itself two bites past the toe. A new suture was begun at this site and run until it met the first suture at the heel of the graft. The second suture was tied to the first at the heel. The bypassed region of artery was ligated. Each arteriotomy was made precisely $6 \mathrm{~cm}$ in length. The arteriotomies were made as slits, with no window of artery removed. Before the anastomoses were constructed, a thin layer of lead oxide was painted on the cut edges of the arteriotomy. In addition, stainless steel wires $0.05 \mathrm{~mm}$ in diameter were placed at the heel, toe, and widest portions of each end-to-side anastomosis. The grafts were perfused in vivo for 30 minutes with pulsatile arterial pressure. Each grafted artery, with both proximal and distal end-to-side anastomoses, was then removed with adjacent arteries. The vessel segments were mounted in vitro and held at in situ length in a tissue bath. The bath and vessels were filled with normal saline solution at $37^{\circ} \mathrm{C}$, and the vessels were pressurized in $25 \mathrm{~mm} \mathrm{Hg}$ steps up to $200 \mathrm{~mm} \mathrm{Hg}$. After several such cycles to relax the vessels, the lumen was again pressurized in $25 \mathrm{~mm} \mathrm{Hg}$ steps to $200 \mathrm{~mm} \mathrm{Hg}$. Anterior-posterior radiographs were obtained at each pressure with a dental radiographic machine and dental film placed immediately beneath the vessel. No contrast material was used because the lead oxide-lined cut edges of artery provided contrast. The radiographs were projected at $\times 22$ magnification, and the major and minor axes of the elliptical anastomoses were determined. The CSAs of the elliptical end-to-side anastomoses were computed according to the following equation:

$$
\mathrm{CSA}=\frac{\pi m M}{4}
$$

where $m$ is the minor axis, or width of the elliptical anastomosis, and $M$ is the major axis, or length of the elliptical anastomosis. The compliance of the end-to-side anastomoses was calculated according to equation 1 , except that the elliptical CSA was used instead of diameter.

All data for the three groups were analyzed statistically with analysis of variance followed by Scheffe's test to compare values between groups. Statistical significance was accepted at the level of $p<0.05$. 


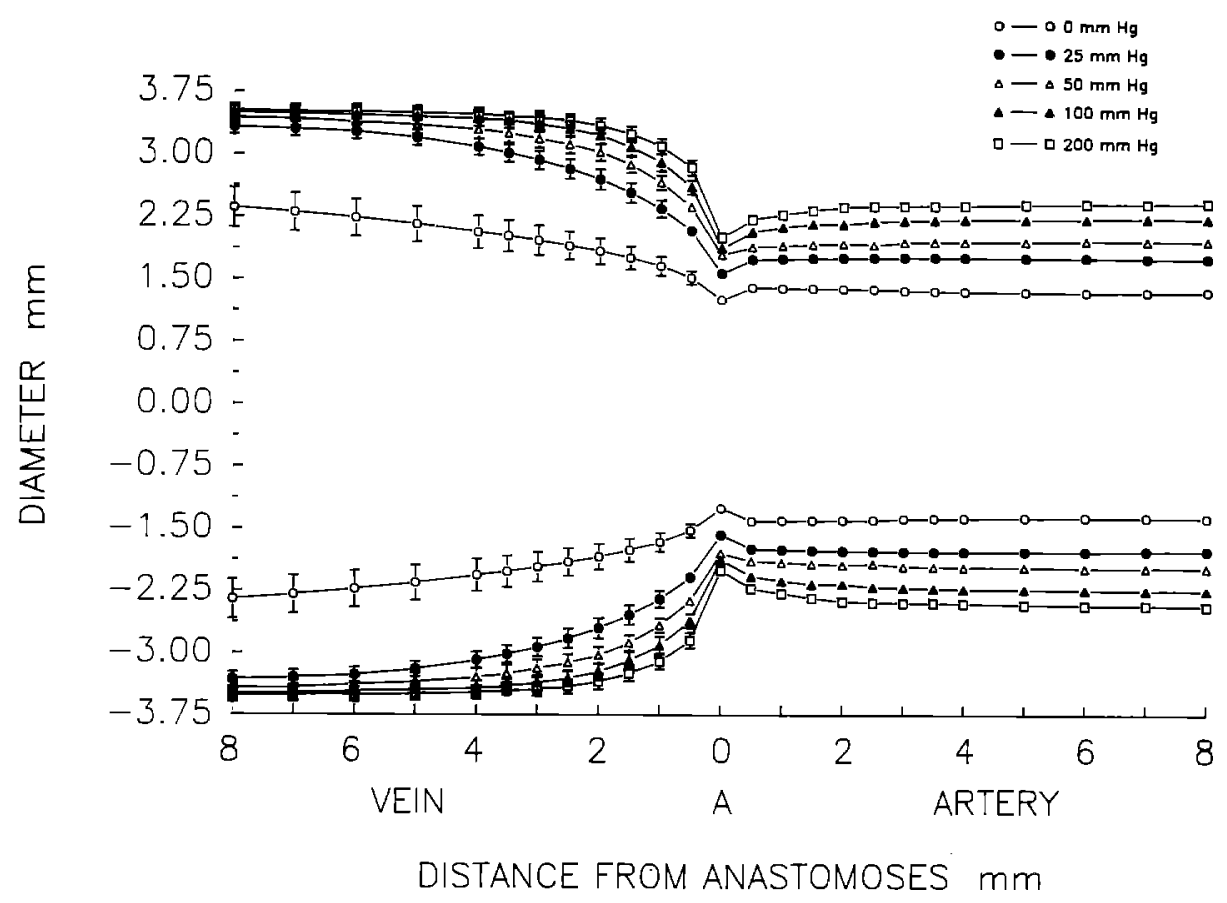

Fig. 2. Diameter isobars for eight end-to-end anastomoses constructed with continuous polypropylene sutures. Data shown are mean \pm standard error of the mean.

\section{Results}

Mechanical properties of suture materials. Fig. 1 shows representative length-force relationships for the two types of suture material. As reflected by the slopes of the curves, at small extensions the polybutester sutures were more extensible than the polypropylene sutures. When stretched about $15 \%$, however, the polybutester sutures became stiffer than the polypropylene sutures. It is important to note that the polybutester sutures were extensible only at small and moderate extensions. The extensibility of polybutester sutures at short extensions results from the biphasic copolymer composition of polybutester. ${ }^{6}$ The breaking force for 17 samples of each suture material averaged $283.1 \pm 10.3 \mathrm{gm}$ (mean \pm standard error of the mean) for the $6-0$ polypropylene sutures and $262.9 \pm 12.5 \mathrm{gm}$ for the 6-0 polybutester sutures. The difference in breaking force between the two suture materials was not statistically significant.

End-to-end anastomoses. The dimensions and mechanical behavior of each type of anastomosis were examined with magnified projections of radiographs obtained at each pressure. Comparison of the anastomoses constructed with interrupted polypropylene and interrupted polybutester sutures at each pressure demonstrated no quantitative or statistical differences between anastomoses with the two different suture materials. The data for the interrupted anastomoses constructed with the two types of suture material were therefore combined into a single group, identified as "interrupted anastomoses." Figs. 2, 3, and 4 present diameter isobars at $0,25,50,100$, and $200 \mathrm{~mm} \mathrm{Hg}$ for the three types of anastomosis. Figs. 2 and 3 show data for eight anastomoses constructed with continuous polypropylene sutures (Fig. 2) and eight constructed with continuous polybutester sutures (Fig. 3). It is evident that the continuous suture lines with both materials caused the anastomosis to act as a restrictive anulus separating the larger vein from the smaller artery. The suture line separated both the dimensions and the considerably different compliance characteristics of the two vessels. Fig. 4 shows data for 10 interrupted suture lines constructed with polypropylene or polybutester sutures. These data show less restriction, with a slight flaring of the artery at the anastomosis at pressures up to $100 \mathrm{~mm}$ 


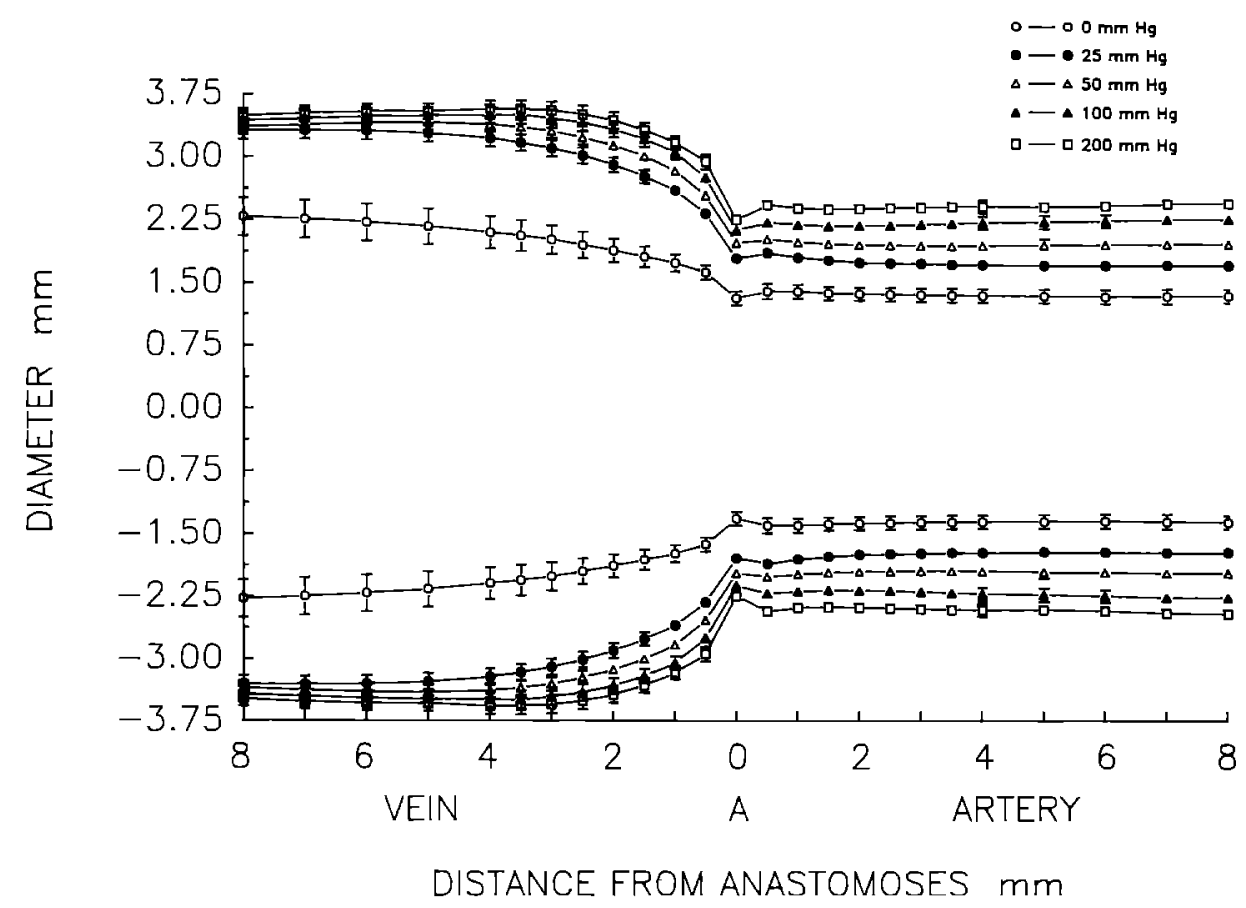

Fig. 3. Diameter isobars for eight end-to-end anastomoses constructed with continuous polybutester sutures. Data shown are mean \pm standard error of the mean.

Hg. This indicates that the interrupted suture line permits the artery to be pulled slightly outward in the radial direction by the larger vein. Although this flaring is largely absent at pressures greater than 100 $\mathrm{mm} \mathrm{Hg}$, it clearly demonstrates that interrupted suture lines do not mechanically separate the two vessels as much as do continuous suture lines. It is also of interest that for all three types of anastomosis the restrictive effects of the anastomoses extended for about $2 \mathrm{~mm}$ along the vein distal to the anastomoses.

Fig. 5 summarizes pressure-diameter curves for the three types of anastomosis. The interrupted anastomoses were the largest, the continuous polybutester anastomoses were next largest, and the continuous polypropylene anastomoses were the smallest. The diameters of the anastomoses were statistically different from one another at all pressures greater than $25 \mathrm{~mm} \mathrm{Hg}(p<0.05)$. It is clear that most of the increase in diameter provided by the continuous polybutester suture was seen at pressures of $50 \mathrm{~mm} \mathrm{Hg}$ or less. This is consistent with the mechanical characteristics of the polybutester suture, which exhibits marked extensibility only at small and moderate loads, and extension up to $15 \%$ (Fig. 1). At pressures greater than $50 \mathrm{~mm}$ $\mathrm{Hg}$, the pressure-diameter curves for the three anastomoses lie parallel to one another, but the polybutester anastomoses remain larger at all pressures.

Table I compares the anastomotic diameters and CSAs of the three end-to-end anastomoses at 100 $\mathrm{mm} \mathrm{Hg}$. All of the anastomotic diameters were significantly different from one another $(p<0.05)$. Table I also gives the CSAs of the anastomoses relative to those of the arteries $6 \mathrm{~mm}$ remote from the anastomoses. The dimensions of the artery are used as a standard. The area of the interrupted anastomoses averaged $107 \%$ of that of the artery. The slight increase was caused by the flaring of the artery at the anastomosis as described previously (Fig. 4). The area of the continuous polybutester anastomoses averaged $90 \%$ of that of the artery, and the area of the continuous polypropylene anastomoses averaged only $70 \%$. Because running polypropylene sutures are often used clinically, Table I also shows the CSAs of the anastomoses relative to those of the continuous polypropylene anastomoses. The 


\section{INTERRUPTED}

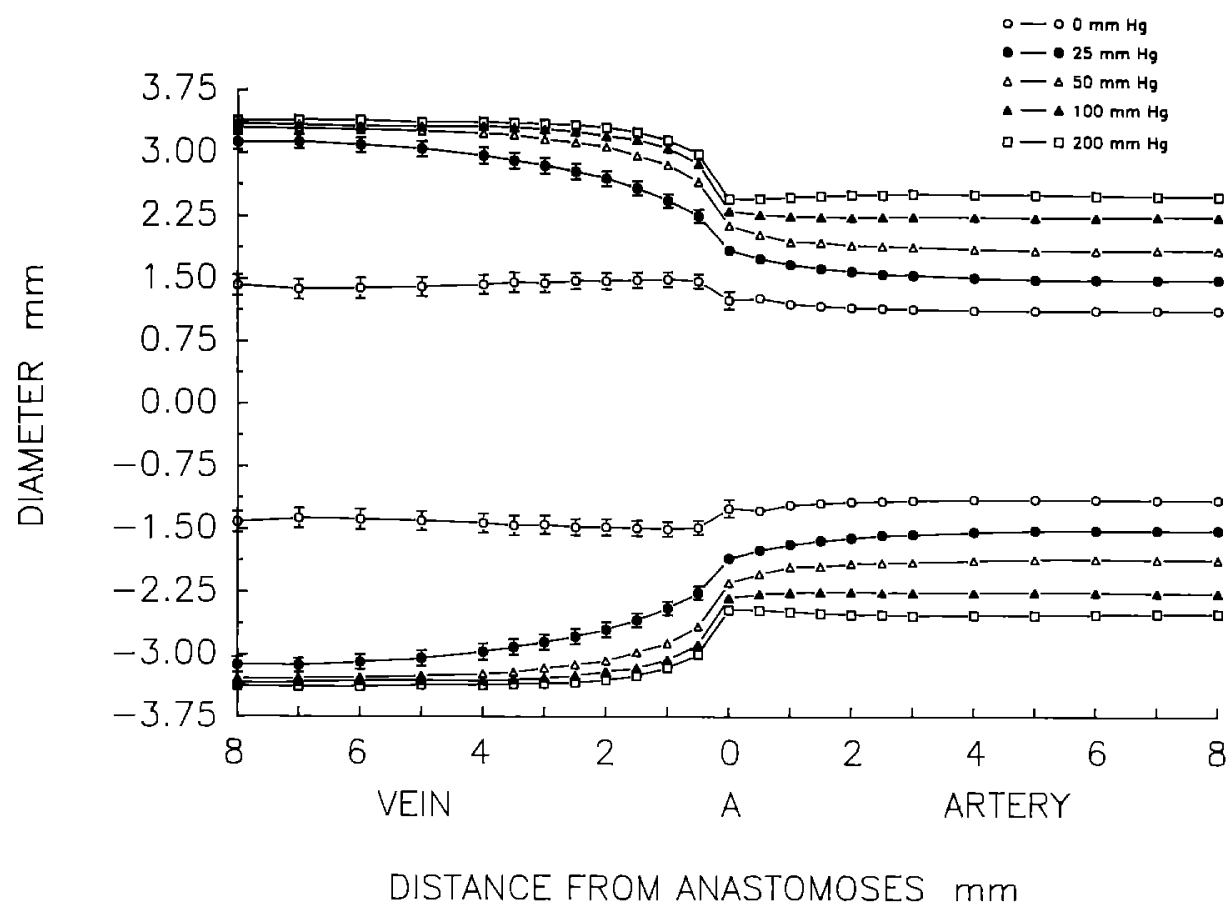

Fig. 4. Diameter isobars for ten end-to-end anastomoses constructed with five interrupted polypropylene and five polybutester sutures.

area of the interrupted anastomoses averaged $153 \%$ of that of polypropylene, and the area of the continuous polybutester anastomoses averaged $128 \%$ of that of polypropylene suture line. It is clear from these data that an interrupted anastomosis is the least restrictive and most closely approximates the dimensions of the native artery, whereas the continuous polypropylene anastomosis is quite restrictive. The continuous polybutester anastomoses appear to be a reasonable compromise, facilitating ease and rapidity of construction with little anastomotic bleeding without providing excessive restriction.

The compliance of the three types of anastomosis was computed according to equation 1 . None of the three types of anastomosis differed statistically in compliance from the others at any location. This was found for all compliances determined at pressures between 50 and $200 \mathrm{~mm} \mathrm{Hg}$. These findings suggest that there is little advantage with respect to compliance in choosing one suture material or suture technique rather than another. They are also consistent with the observation that most of the compliance of the polybutester suture is present only at small and moderate extensions. Nevertheless, the extensibility at small extensions is important; as shown by Table I, this suture extensibility at small loads permits dilation of the suture line, thereby conferring a larger end-to-end anastomosis.

End-to-side anastomoses. Fig. 6 presents pressure-major axis (length) curves for 10 interrupted, 16 continuous polybutester, and 16 continuous polypropylene anastomoses. At $0 \mathrm{~mm} \mathrm{Hg}$, the length of the anastomoses constructed with continuous polypropylene sutures remained larger than those constructed with continuous polybutester or interrupted sutures. This occurred because the polypropylene anastomoses, when not loaded by pressure, were prevented from retracting by the stiffness of the suture material. In contrast, the anastomoses constructed with running compliant polybutester or interrupted sutures were able to retract. When pressurized to arterial pressures, however, the three types of suture lines achieved similar lengths. None of the anastomotic lengths differed significantly from the others at $100 \mathrm{~mm} \mathrm{Hg}$. Fig. 7 presents pressure-minor axis (width) curves for the same 


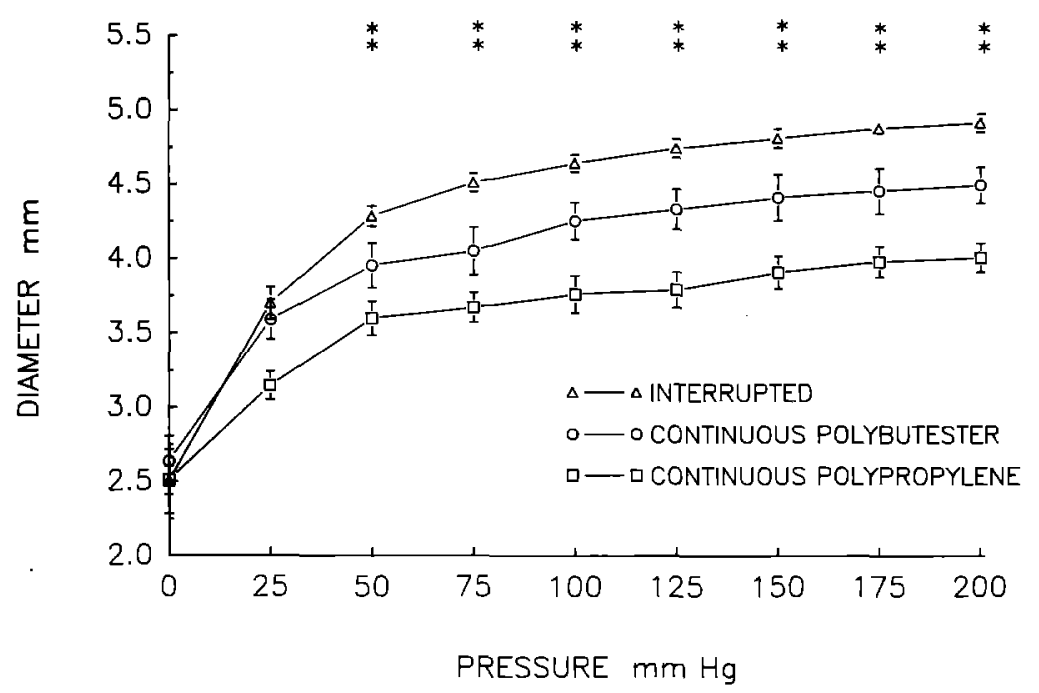

Fig. 5. Pressure-diameter curves for end-to-end anastomoses. Data are shown for 10 interrupted anastomoses, eight continuous polybutester anastomoses, and eight continuous polypropylene anastomoses. Double asterisk indicates significant differences between all three curves.

Table I. Dimensions of end-to-end anastomoses at $100 \mathrm{mg} \mathrm{Hg}$ pressure

\begin{tabular}{lcccc}
\multicolumn{1}{c}{ Artery } & Anastomoses & $\begin{array}{c}\text { Internipted } \\
\text { anastomoses }\end{array}$ & $\begin{array}{c}\text { Continuous polybutester } \\
\text { anastomoses }\end{array}$ & $\begin{array}{c}\text { Continuous polypropylene } \\
\text { anastomoses }\end{array}$ \\
\hline Diameter $(\mathrm{mm})$ & 4.49 & 4.65 & 4.26 & 3.76 \\
CSA (mm $\left.{ }^{2}\right)$ & 15.83 & 16.98 & 14.25 & 11.10 \\
CSA (\% of artery) & 100 & 107 & 90 & 70 \\
CSA (\% of continuous polypropylene & 143 & 153 & 128 & 100 \\
$\quad$ anastomosis) & & & \\
\hline
\end{tabular}

three sets of anastomoses. The curves for the three types of anastomosis were remarkably similar and did not differ statistically significantly from one another at any pressure. Finally, Fig. 8 presents pressure-CSA curves for the three end-to-side anastomoses. Again, there were no statistical differences in CSA among the three types of anastomoses except at $200 \mathrm{~mm} \mathrm{Hg}(p<0.05)$. Unlike the very different diameters and areas obtained in the endto-end anastomoses with different suture techniques (Fig. 5, Table I), the end-to-side anastomoses were almost identical in circumference and CSA with the different suture materials and different suture techniques.

The CSAs of the three types of anastomoses were used to compute the compliance of the elliptical end-to-side anastomoses. The compliances of the three types of end-to-side anastomosis were virtually identical, with no statistically significant differences at any pressure.

\section{Discussion}

These experiments examined the mechanics of anastomoses constructed with native artery and somewhat larger autologous vein. Oversized vein was used to avoid limiting the dimensions or properties of the anastomoses by the size of the graft. The data shown in Figs. 2 through 5 for end-to-end anastomoses demonstrate that the dimensions and mechanical characteristics of these anastomoses are determined by the properties of the suture material and the suture technique used and are not limited by the properties of the artery or the vein graft. In contrast, the data shown in Figs. 7 and 8 for end-to-side anastomoses demonstrate that the dimensions and mechanical characteristics of these 


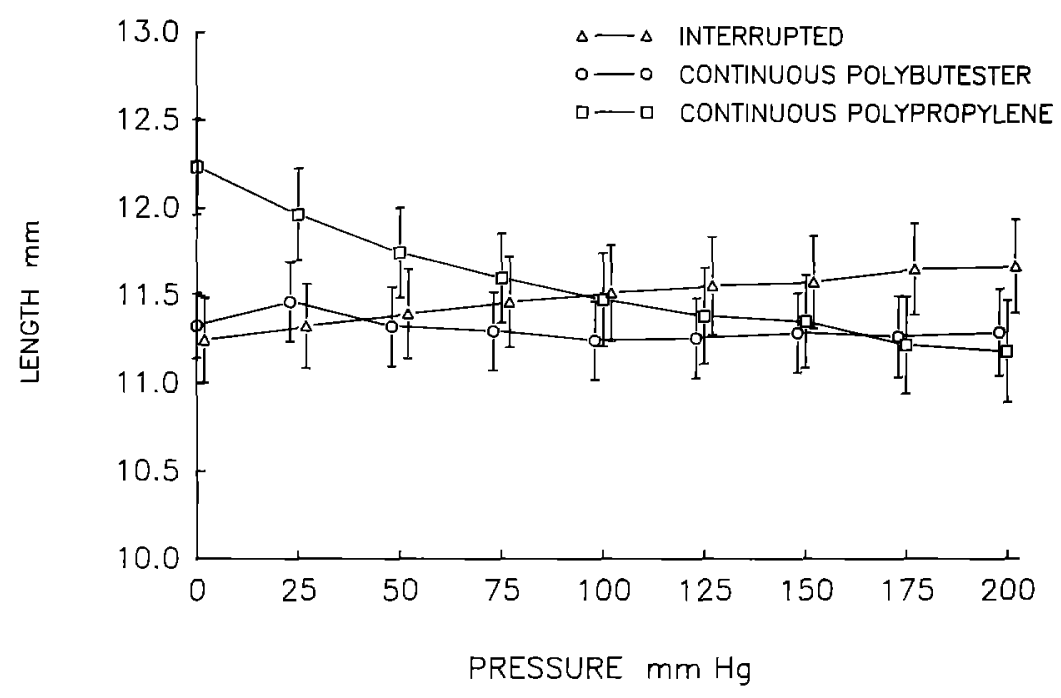

Fig. 6. Pressure-length relationships for 42 end-to-side anastomoses. Curves were significantly different only at $0 \mathrm{~mm} \mathrm{Hg}$.

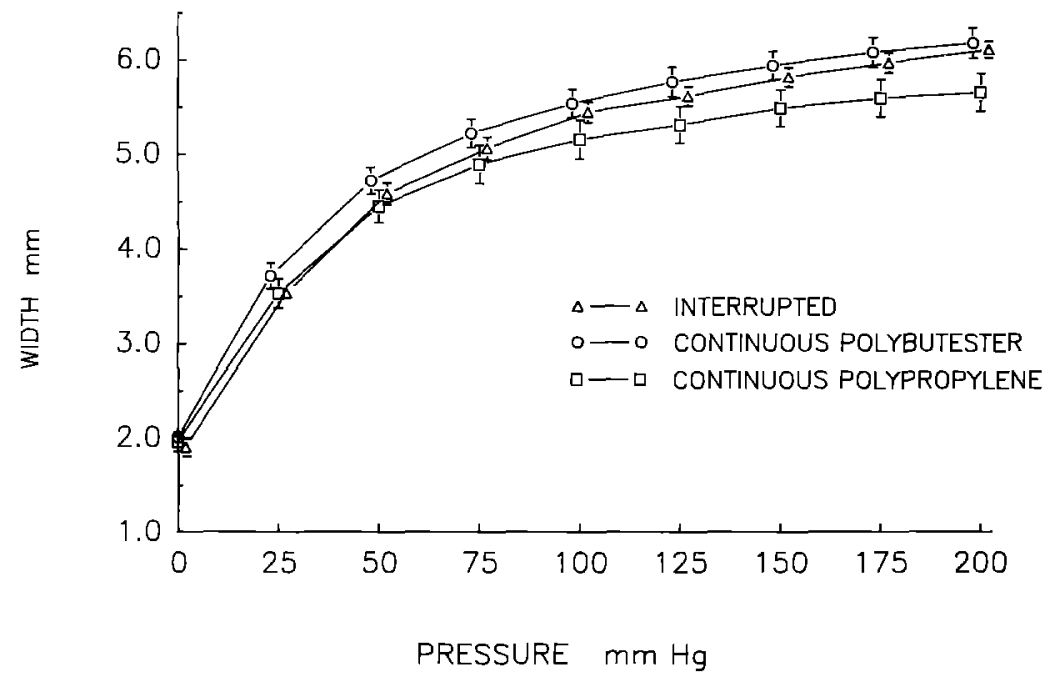

Fig. 7. Pressure-width relationships for 42 anastomoses. Curves were not significantly different from one another at any pressure.

anastomoses are determined largely by the mechanical properties of the adjoining vessels, especially of the native artery. The artery exhibits almost no change in length with pressure ${ }^{3,8}$ but does tend to shorten slightly (Fig. 6) as the width of the anastomosis is increased by pressure (Fig. 7). The veins used for the end-to-side anastomoses were larger than the arteries and therefore were not critical 


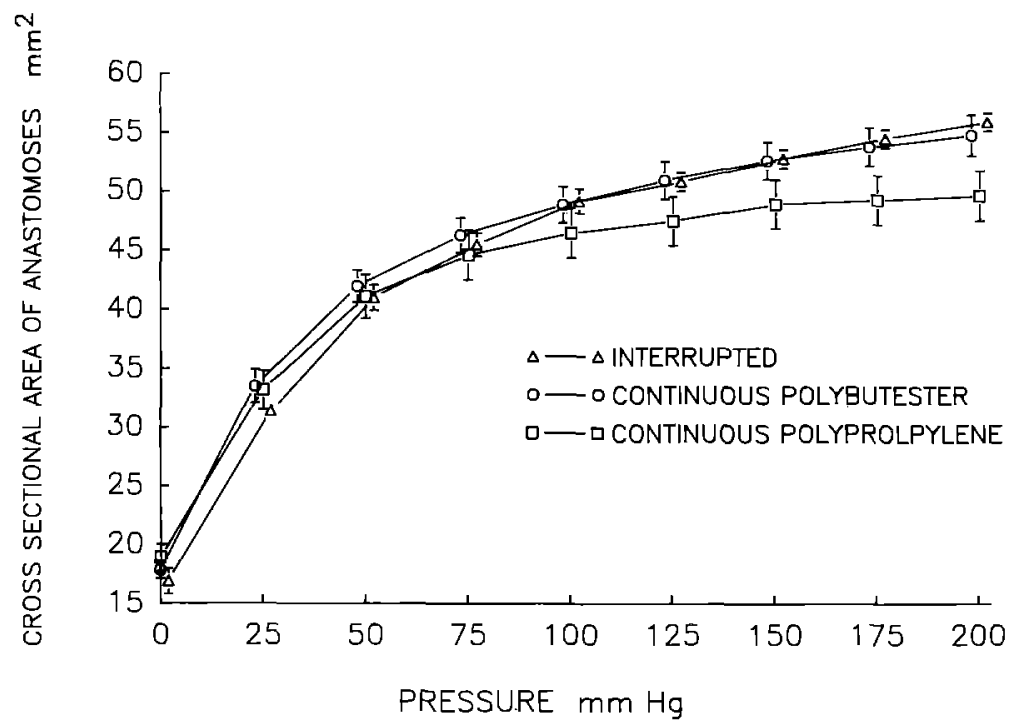

Fig. 8. Pressure-CSA curves of the 42 end-to-side anastomoses. Only continuous polypropylene anastomoses were significantly different $(p<0.05)$, and only at $200 \mathrm{~mm} \mathrm{Hg}$.

determinants of anastomotic dimensions. It should be emphasized that these findings apply only to arteriovenous anastomoses. They must be applied with caution to arterioarterial or venovenous anastomoses, and they certainly cannot be applied to anastomoses with rigid prosthetic grafts.

In 1960, Szilagyi and coworkers ${ }^{9}$ demonstrated that end-to-end and end-to-side anastomoses can be made to deliver nearly equal volumes of flow if end-to-side anastomoses are constructed with a small graft-artery angle and if a small window is removed from the recipient artery to enhance the anastomotic lumen. Ideally, the drop in pressure and volume of flow delivered should not be restricted by grafts or anastomoses but rather by downstream resistance. ${ }^{3}$ The experiments presented here show that for end-to-end anastomoses the least restrictive anastomosis was produced by an interrupted suture technique with either polypropylene or polybutester suture. The next largest anastomosis was produced by a continuous anastomosis with polybutester, a suture material that is extensible at moderate to low extensions (Fig. 1). The most restrictive anastomosis was that produced with continuous polypropylene, a suture material that is stiff at all degrees of extension (Fig. 1). These data suggest that when construct- ing an end-to-end anastomosis one should consider both suture technique and suture material selection. In the case of small vessels, such as the renal and mesenteric arteries, an interrupted technique should be used to maximize the area of the anastomosis. Another solution may be to use a spatulated or beveled anastomosis. In the case of larger vessels, the technique of constructing the anastomoses is not as critical because of the large diameter and high-volume flow. In this case, an interrupted anastomosis is impractical because of the time necessary to construct it and because of bleeding from the suture line. A compromise may be achieved with a continuous polybutester suture, however, because this provides a significantly larger anastomoses than one constructed with a continuous polypropylene suture (Table I).

\section{REFERENCES}

1. Klein SR, Goldberg L, Miranda RM, et al. Effect of suture technique on arterial anastomotic compliance. Arch Surg 1982;117:45-7.

2. Fillinger MF, Kerns B, Bruch D, et al. Does the end-to-end venous anastomosis offer a functional advantage over the end-to-side venous anastomosis in high-output arteriovenous grafts? J Vasc Surg 1990;12:676-90.

3. Dobrin PB, Canfield TR, Moran J, et al. Coronary artery bypass: the physiological basis for differences in flow with 
internal mammary artery and saphenous vein grafts. J Thorac Cardiovasc Surg 1977;74:445-54.

4. LoGerfo F, Soncrant T, Teel T, et al. Boundary layer separation in models of side-to-end arterial anastomoses. Arch Surg 1979;114:1369-73.

5. Dobrin PB. Mechanical factors associated with the development of intimal hyperplasia with respect to vascular grafts. In: Dobrin PB, ed. Intimal hyperplasia. Austin: RG Landes, 1994:85-109.

6. Rodeheaver GT, Borzelleca DC, Thacker JG, et al. Unique performance characteristics of Novafil ${ }^{\mathrm{E}}$. Surg Gynec Obstet 1987;164:230-6.

7. Rodeheaver GT, Nesbit WS, Eddich RF. Novafil ${ }^{\text {(i) }}$ : a dynamic suture for wound closure. Ann Surg 1986;204:193-9.

8. Dobrin PB. Vascular mechanics. In: Handbook of physiology. Part I. Peripheral circulation and organ blood flow, vol 3. Baltimore: Williams and Wilkins, 1983:65-102.

9. Szilagyi DE, Whitcomb J, Schenker W, et al. The laws of fluid flow and arterial grafting. Surgery 1960;47:55-67. 